\title{
Comparing the onset of maxillary infiltration local anaesthesia and pain experience using the conventional technique vs. the Wand in children
}

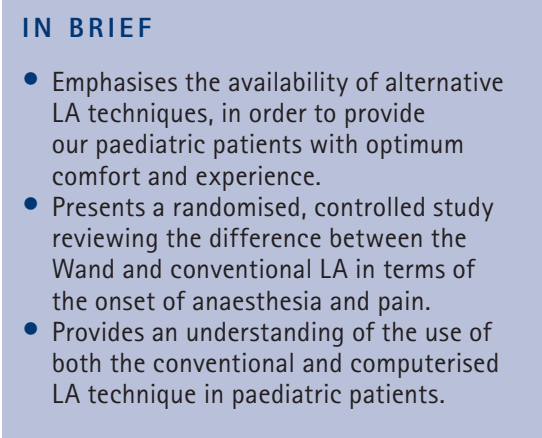

\author{
P. Kandiah ${ }^{1}$ and J. F. Tahmassebi²
}

\begin{abstract}
Aim This prospective, randomised, parallel, controlled study was conducted firstly to compare the onset of local anaesthesia (LA) when using the conventional technique versus the Wand computer-controlled LA and secondly to assess the pain experience in children. Method Thirty children were randomly allocated to the treatment group (Wand) or the control group (conventional). Lidocaine $2 \%$ with adrenaline $(1: 80,000)$ was given as a buccal infiltration. The onset of pulpal anaesthesia was tested using an analytic electric pulp tester (EPT). The pain experience during the LA was recorded using a modified visual analogue score (VAS). Results Median time for the onset of LA was 6.30 minutes for the control and 7.25 minutes for the Wand group. Mean pain experience score for the control group was 9.78\% as opposed to $8.46 \%$ in the Wand group. Statistical analysis showed that there was no statistically significant difference in the onset of LA ( $p=0.486)$ and the pain experience ( $p=0.713$ ) between the two groups. Conclusion When placing a buccal infiltration on upper first permanent molars, the onset of LA and the pain experience was no different using the Wand and the conventional technique.
\end{abstract}

\section{INTRODUCTION}

Pain and anxiety related to dentistry have historically been the main reason for poor attendance at the dentist. ${ }^{1}$ The administration of local anaesthesia (LA) for children can be a challenge and clinicians are often confronted by patients who find LA the most stressful part of their treatment. A rapidly acting, pain-free LA system is the gold standard ideal, especially in paediatric patients.

Pain behaviour and distress in children have been investigated in studies comparing the Wand LA and conventional LA. ${ }^{2}$ The first reported study in the literature on the use of the Wand in children was in 1999 by Asarch et al. ${ }^{3}$ The findings of this study showed no significant difference between Wand LA and the conventional technique. However, in this study, pain behaviour during three different types of LA including inferior alveolar block, palatal infiltration

${ }^{1 *}$ Specialist Registrar in Paediatric Dentistry, ${ }^{2}$ Senior Lecturer in Paediatric Dentistry, Department of Paediatric Dentistry, Leeds Dental Institute, Clarendon Way, Leeds, LS2 9LU

${ }^{*}$ Correspondence to: Dr P. Kandiah

Email:anjalikandiah@hotmail.com

Online article number E15

Refereed Paper - accepted 6 July 2012

DOI: $10.1038 /$ sj.bdj.2012.988

${ }^{\oplus}$ British Dental Journal 2012; 213: E15 and buccal infiltration was reviewed. The duration of LA delivery was not standardised. In a subsequent study in children aged 5-13 years old by Gibson et al. ${ }^{4}$ these problems were addressed. The Wand was indeed found to produce significantly less disruptive behaviours during the initial $15 \mathrm{sec}-$ onds of the injection. Allen et al. ${ }^{5}$ supported these findings in their research. However, the investigators questioned whether Wand LA delivery was suitable for preschool children as they found that prolonged duration of this method was likely to result in more restless behaviour.

Palm et al. investigated the onset of LA following an inferior dental nerve block or IDB. ${ }^{6}$ This was a split mouth, randomised trial using the Wand and the conventional anaesthesia system. The onset of LA and pain perception in children and adolescent patients was evaluated. The subjects were aged between 7 and 18 years old. The onset of LA following an IDB was assessed using patients perception of 'numbness' and a ten point visual analogue scale to assess pain experience. The design of this study did not allow for an objective measure of the onset of LA and instead relied on the patients' perception.

From the literature review, it is apparent that there is a lack of literature comparing the onset of local anaesthesia using different techniques. Lee et $a .^{7}{ }^{7}$ carried out their study to assess the efficacy of the anterior middle superior alveolar injection, comparing the Wand and conventional LA. Statistical analysis of the onset and duration of anaesthesia was not performed due to the lack of numbers.

In this study, the primary aim was to carry out a prospective, randomised, parallel, controlled study to evaluate and compare the onset of LA when using conventional technique (control group) $v s$. the Wand (treatment group) for buccal infiltrations. The secondary aim was to assess the pain experience between the two techniques.

\section{NULL HYPOTHESIS}

\section{Primary null hypothesis}

There is no difference in the onset of maxillary infiltration local anaesthesia between the conventional technique $v s$. the Wand in children

\section{Secondary null hypothesis}

There is no difference in the pain experience of maxillary infiltration local anaesthesia between the conventional technique $v s$. the Wand in children. 


\section{MATERIALS AND METHODS}

The present study was carried out between October 2009 and May 2010 and was conducted at two sites, the paediatric department at the Leeds Dental Institute and the Community Dental Clinic in Barnsley. Ethical approval was obtained from Northern and Yorkshire REC and Research and development approval was gained from Leeds Teaching Hospital Trust and Barnsley RetD.

\section{Materials}

The materials required for this study included a dark pair of sunglasses for the patient, a stop clock to record the onset of LA and an analytic electric pulp tester (SybronEndo Vitality scanner, Click Dental Supplies Ltd, Barn Mews, Dunton Road, Basildon, Essex, SS15 4DB). KY jelly was required to act a medium for the scanner. The topical LA was 20\% benzocaine ultracare bubble gum flavoured gel (Optident, International Development Centre, Valley Drive, Ilkley, West Yorkshire, LS29 $8 \mathrm{~PB})$. The LA used was the $1.8 \mathrm{ml}$ cartridge of Lignospan (Lignospan Sepcial 2\% lidocaine with 1:80,000 adrenaline). The manufactures of the LA were Septodont (Unit RetS, Orchard Business Centre, St Barnabas Close, Allington, Maidstone, Kent ME16 0JZ). The study also required the Wand LA system (DPS, 6 Ayot St Lawrence, Welwyn, Herts, AL6 9BP) and conventional syringes (NHS supply chain, West Way, Coles Park Industrial Estates, Alfreton, Derbyshire, DE55 4QJ).

\section{Power calculations}

The power calculation was based on a power of 90\% and a significance level of 5\%. The C value is 10.507 Snedecor and Cochrane 1989 (Dell et al.). ${ }^{8}$ Minimal detectable difference will be set at $60 \mathrm{~s}$ and standard deviation at $30 \mathrm{~s}$.

\section{Basic formula for sample size calculation}

$\mathrm{N}=1+2 \times \mathrm{C}(\mathrm{S} / \mathrm{D})^{2}$ (Dell et al. $)^{8}$.

Where $\mathrm{C}$ is a constant depends on the significance level $\alpha$ and $\beta$.

$\mathrm{S}=$ Standard deviation

$\mathrm{D}=$ Minimal difference you want

to detect

Therefore using the above formula:

$\mathrm{N}=1+2 \times 10.507(30 / 60)^{2}=6.2535$

\section{Instructions: Please mark on the line how you felt about this injection.}

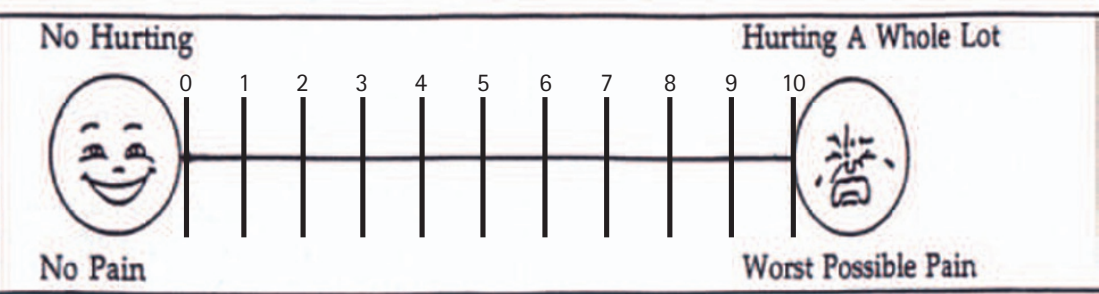

Fig. 1 The modified visual analogue scale (VAS)

\begin{tabular}{|c|c|c|c|c|c|c|}
\hline $\begin{array}{l}\text { No of } \\
\text { participants }\end{array}$ & Median & $\begin{array}{l}\text { Standard } \\
\text { deviation }\end{array}$ & Mean & Minimum & Maximum & Range \\
\hline 30 & 12.00 & 2.177 & 11.87 & 8 & 16 & 8 \\
\hline
\end{tabular}

Table 2 Sex distribution of the study population

\begin{tabular}{l|l|l} 
& Frequency & Percentage (\%) \\
\hline Female & 19 & 63.3 \\
\hline Male & 11 & 36.7 \\
\hline Total & 30 & 100
\end{tabular}

\begin{tabular}{|c|c|c|c|c|c|c|c|}
\hline Groups & $\begin{array}{l}\text { No of } \\
\text { participants }\end{array}$ & Mean & Median & $\begin{array}{l}\text { Standard } \\
\text { deviation }\end{array}$ & Minimum & Maximum & Range \\
\hline Control & 15 & 2.096 & 2.120 & 0.515 & 1.39 & 3.40 & 2.01 \\
\hline Treatment & 15 & 2.266 & 2.200 & 0.602 & 1.53 & 4.21 & 2.68 \\
\hline Total & 30 & 2.181 & 2.145 & 0.557 & 1.39 & 4.21 & 2.82 \\
\hline
\end{tabular}

Therefore the sample size was calculated to be six in the control and six in the treatment group (total of 12). In order to adjust for possible drop-outs, as well as the pilot study, it was decided to have 15 in each group.

\section{Sample size}

Following the power calculation and the adjustments made after advice from the statistician it was decided to have 30 patients in total for the study (15 in the treatment group and 15 in the control). In addition, a pilot study was carried out before the main study including four patients in total (two control and two for the treatment group).

\section{Inclusion criteria}

Patients aged 8-16 years old, who were graded I according to the American
Society of Anaesthesiologist (ASA) physical status classification were selected for the study (http://www.asahq.org/clinical/ physicalstatus.htm). Only patients who required restoration of upper first permanent molars with minimal carious lesions (less than 1/3 marginal ridge involved or small occlusal caries) who were asymptomatic and without any associated sinus or pathology were included in this study. Caries diagnosis was made by clinical and radiographic examination and this was concurred by an independent specialist in paediatric dentistry.

\section{Exclusion criteria}

Individuals who were unable to communicate or had a significant needle phobia and required additional use of conscious sedation were not included in the study. Patients with heavily restored dentition 
or teeth with enamel/dentinal defect were also excluded from the study. Inability to obtain a positive baseline reading using the electric pulp tester was also a reason to exclude participants from the study as well as the inability to obtain positive consent from parents or guardian.

\section{Randomisation}

The children were randomly assigned into either the treatment group or the control group. The statistician carried out the randomisation by block allocation, based on a random table of numbers, according to a computer programme of random allocation (http://randomisation.com).

The randomisation data was sent to the specialist in paediatric dentistry in Barnsley CDS (RM) while the investigator remained blind. The random allocations were placed into envelopes by RM who then held the envelopes that were only given to the investigator when the patient arrived for treatment. In the case of patients who were seen at the LDI, RM would provide the appropriate envelope(s) in advance. The envelope would only be opened by the investigator immediately before the LA. In this study, although the patient was blind to the LA given, the single operator could not be blinded for the practical purposes of LA delivery and in order to measure the outcomes.

\section{Pilot study}

Before the start of the study, the patient information sheet was piloted at Barnsley for feedback. The information sheet was written based on the understanding and reading ability of children aged 8-16 years old. It was commented by one child, that having numbers on the modified VAS would help with scoring. Following feedback the VAS scale was modified accordingly to make it more user friendly, for example, subdividing the line up from 0-10 to grade the level of pain.

\section{Procedure}

An appropriately trained, qualified nurse placed a bib and a dark pair of sunglasses onto the child to minimise the child being able to see the LA. The pulp tester was used on the upper first permanent molar that was to be anaesthetised. This was to establish a baseline reading.
The dialogue for the technique was standardised for both the Wand and the control group as follows:

'I am going to put some bubble gum gel on your gum to help the gum go to sleep.'

'I am now going to spray some juice to help the tooth to go numb, I will keep the straw in your mouth to stop any juice spilling into your mouth.'

The wand's bleeping system was an indicator of LA delivery. To avoid this being a potential source of bias, it was planned that the beeping sound would be used for both groups of patients. The LA used was $1.8 \mathrm{ml}$ cartridge of Lignospan special containing lidocaine hydrochloride $2 \%$ and adrenaline (adrenaline) 1:80,000. Topical LA was placed for two minutes against the dry gingival of the first permanent molar using a cotton wool roll. On penetrating the mucosa with the needle, the nurse started the stop clock. On completion of the administration of the LA (total of $1.8 \mathrm{ml}$ ) the time taken to deliver the LA was recorded by the nurse. For both groups a $1.8 \mathrm{ml}$ cartridge was used. The pulp tester was then placed on the tooth immediately after the deposition of the LA. Pulp tester reading was repeated every 60 seconds until the patient was unable to sense the impulses (two subsequent readings of 80 ) and hence this was regarded as the end point. The nurse recorded the number of 60 second cycles. The patient was then asked to indicate on the scale provided the pain experience during the administration of the LA. The modified VAS scale was a $100 \mathrm{~mm}$ scale with descriptive anchors at each end as shown on Figure 1. The pain score was calculated by measuring the distance in millimetres of the mark from the left end. The VAS scales were divided into percentages: $10 \mathrm{~mm}$ indicating 10\%, $20 \mathrm{~mm}$ indicating $20 \%$ etc. The estimation of pain was as follows: $0-20 \%=$ no pain, $21-40 \%=$ mild pain, $41-60 \%=$ moderate pain, $61-80 \%=$ severe pain and $81-100 \%=$ intolerable pain.

\section{Statistical analysis}

The data was collected prospectively with the help of the nurse on the data sheet. The data was input into the SPSS database. All data was stored on a password encrypted USB memory stick.

The data collected was analysed using SPSS version 16 . The data related to the onset of LA and pain experience was summarised using medians. To check for normal distribution, both sets of data were analysed using One Sample Kolmogorov Smirnov Test on SPSS. Mann Whitney U-Test was used to carry out the hypothesis testing.

\section{RESULTS}

The study was carried out at two sites, the Leeds Dental Institute (LDI), Leeds and the Community Dental Service (CDS), Barnsley. The majority of the subjects for the study were recruited from Barnsley (83\%). Altogether 34 potentially suitable subjects were approached to take part in the study, aged between 8 and 16 years old with a median age of 12 years (standard deviation \pm 2.177 ). Three cases were abandoned due to problems associated with the electric pulp tester (EPT). Out of the three, one patient started crying when the EPT was used and for the others the EPT response was unreliable. The parents of one patient did not consent for their child to take part in the study.

Children were chosen from the age range 8-16 years old to ensure that first permanent molars had fully erupted and that these children could respond to EPT reliably (Table 1).

The study population consisted of 19 females and 11 males (Table 2). Within the control group there were 8 female and 7 male subjects. In the treatment group, however, there was a bigger difference with 11 female and 4 male participants.

\section{Time taken for local anaesthesia delivery}

The time taken to deliver LA can be variable. The delivery of LA with the Wand is foot pedal controlled and there are two fixed speeds. The conventional technique delivery of LA is operator dependent. Both techniques were adopted and used in the recommended way by a single operator.

The findings from this study (Table 3) suggest that the median for both groups was approximately the same (treatment group 2.12 and control 2.2 minutes). The range appears greater in the treatment group (2.68 minutes) than the control (2.01 minutes), which is likely to be the result of the outliers shown in Figure 2. However, the general pattern of the results 
shows that the spread is generally broader for the control group as opposed to the computerised LA delivery system of the treatment group.

\section{Onset of local anaesthesia}

The onset of anaesthesia or end point was determined by having two consecutive readings of 80 on the EPT from which the first 80 reading was defined as the onset of LA (Table 4). Out of the 30 children involved in the study, three participants did not reach the end point using the EPT. These subjects were excluded from this data analysis for the onset of LA. All three were from the treatment group. Although the EPT was used to establish anaesthesia, it is crucial to mention that in all three cases, treatment was carried out successfully without additional LA. This would suggest that anaesthesia was achieved despite not reaching the end point.

The results from this study showed that the median time taken for the onset of LA was 6.3 minutes for the control group and 7.2 minutes for the treatment group. Figure 3 shows, that although the median is approximately the same for both groups, there appears to be a wider spread in the treatment group than the control.

\section{Pain experience-modified visual analogue score}

The majority of the patients from both groups felt that there was no pain involved during the administration of the LA as shown in Table 5. The treatment group had marginally more patients (14/15) expressing that no pain at all was experienced as opposed to the control group (12/14).

\section{DISCUSSION}

This study showed that there was no difference in the onset of local anaesthesia or pain difference when using the Wand and conventional technique when administering buccal infiltration in children. The outcome of no difference in the onset of local anaesthesia is consistent with the findings of Palm et al. ${ }^{6}$ who investigated the onset following an inferior alveolar nerve block.

In this study, the age range was between 8-16 years old. The age distribution for the control group was from 8-14 years old and for the treatment group it was from 9-16 years old. Although the authors were aware that the wide age range could

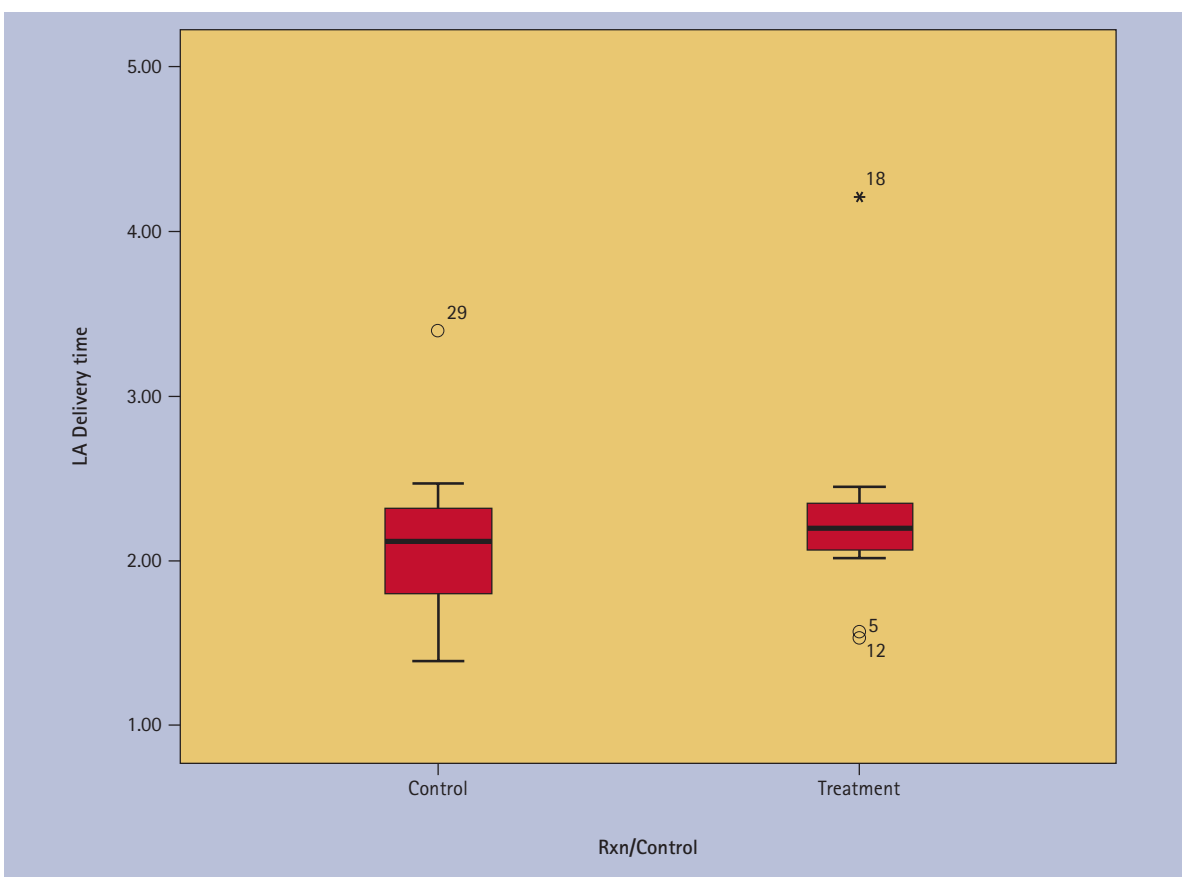

Fig. 2 Time taken to deliver local anaesthesia

\begin{tabular}{|c|c|c|c|c|c|c|c|}
\hline Groups & $\begin{array}{l}\text { No of } \\
\text { participants }\end{array}$ & Mean & Median & $\begin{array}{l}\text { Standard } \\
\text { deviation }\end{array}$ & Minimum & Maximum & Range \\
\hline Control & 15 & 6.053 & 6.300 & 2.681 & 3.00 & 12.00 & 9.00 \\
\hline Treatment & 12 & 6.760 & 7.250 & 3.036 & 3.05 & 12.85 & 9.80 \\
\hline
\end{tabular}

Table 5 Modified VAS results

\begin{tabular}{l|l|l|l|l}
\multirow{2}{*}{ Group } & \multicolumn{2}{|l|}{ Pain (\%) } & Mild & Moderate \\
\cline { 2 - 5 } & No pain & 1 & 2 & 15 \\
\hline Control & 12 & 0 & 1 & 15 \\
\hline Treatment & 14 & 1 & 3 & 30 \\
\hline Total & 26 & & 30 \\
\hline
\end{tabular}

impact on the results, particularly in terms of the patients' perception of pain, all patients chosen were found to be suitable in terms of their understanding as well as their maturity. The sex distribution was approximately the same in the control group; however, in the treatment group there were more females than males. The majority of the previous studies carried out on children were not sex matched., ${ }^{4,5}, 9$

Within the study design, all patients were chosen according to the selection criteria and pre-assessed by the operator. This gave the opportunity to provide patients and parents with information related to the study and also to gain consent. During the pre-assessment, all patients had an introductory appointment to LA including the introduction to topical LA. This was felt to be important, as past dental experience could not be standardised. During the appointment, it was agreed that parents should be present at all times to act as a passive observer. ${ }^{10}$

This study took into careful consideration many of the possible sources of bias. Randomisation by block allocation of the patients was employed to ensure that there was no selection bias. In the study by Tahmassebi et al. ${ }^{11}$ blinding of the patients was not carried out, however, single blinding was successfully carried out in this study. All measures were employed to standardise the dialogue with the patient and to ensure that the 'bleeping' noise of the Wand was replicated for the control group. 


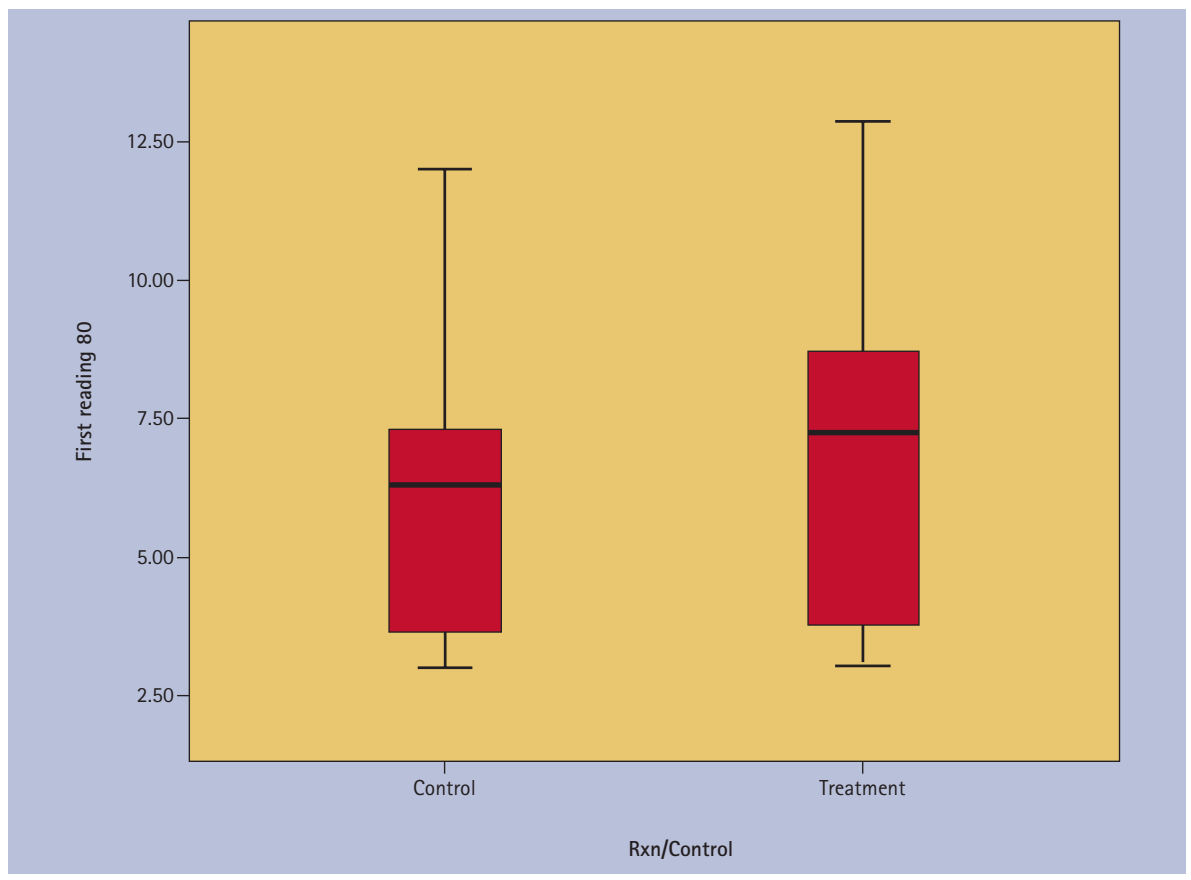

Fig. 3 Onset of local anaesthesia

Despite anecdotal evidence, which suggests that the Wand delivers LA more slowly than the conventional technique, this study showed the time taken to deliver LA was similar in both groups. The median time to deliver $1.8 \mathrm{ml}$ of lignospan LA was 2.12 minutes in the control group and 2.20 in the treatment group. As predicted the range is smaller for the treatment group compared to the control group.

In the majority of studies looking at the onset of local anaesthesia, an EPT was used. Admittedly, most of these studies were carried out on adult patients. In this study, the EPT did show some inconsistencies. Three patients expressed pain on using the EPT and a number of children complained of discomfort. One patient, who was unable to cope, started crying on baseline EPT reading due to pain. From this study, the onset of LA was shown to be similar in both the control and in the treatment group. Median time for the onset of LA was 6.3 minutes for the control compared with 7.25 minutes in the treatment group. The results are in agreement with the findings of Palm et al. in studies have suggested that the Wand was less painful on needle insertion or the injection overall compared with the conventional technique. ${ }^{9,13}$

\section{CONCLUSION}

The authors found no difference in the onset of LA and the pain experience using the Wand and the conventional technique when placing a buccal infiltration on upper first permanent molars.

Gratitude goes to the Leeds Dental Institute and Barnsley Community Dental Service for their help and support during the study and Theresa Munyombwe who helped with all statistics in this project.

1. Hochman M, Chiarello D, Hochman C B, Lopatkin R, Pergola S. Computerized local anaesthesia vs. traditional syringe technique: Subjective pain response. N Y State Dent J 1997; 63: 24-27.

2. Versloot J, Veerkamp J S, Hoogstraten J. Pain behaviour and distress in children during two sequential dental visits: comparing a computerised anaesthesia delivery system and a traditional syringe. $\mathrm{Br}$ Dent J 2008; 205: E2, discussion 30-31.

3. Asarch T, Allen K, Petersen B, Beiraghi S. Efficacy of a computerized local anaesthesia device in pediatric dentistry. Pediatr Dent 1999; 21: 421-424.

4. Gibson R S, Allen K, Huftless S, Beiraghi S. The Wand vs. traditional injections: a comparison of pain related behaviours. Pediatr Dent 2000; 22: 458-462.

5. Allen K D, Kotil D, Larzelere R E, Hutfless S, Beiraghi S. Comparison of a computerized anaesthesia devise with a traditional syringe in preschool children. Pediatr Dent 2002; 24: 315-320.

6. Palm A M, Kirkegaard U, Poulsen S. The wand versus traditional injections for mandibular nerve block in children and adolescents: perceived pain and time of onset. Pediatr Dent 2004; 26: 481-484.

7. Lee S, Reader A, Nusstein J, Beck M, Weaver J. Anesthetic efficacy of the anterior middle superior alveolar (AMSA) injection. Anesth Prog 2004; 51: 80-89.

8. Snedecor GW, Cochran WG. 1989. Statistical Methods. 8th Ed. Ames: lowa State Press; Dell R B. Holleran S, Ramakrishnan R. Sample size determination. ILAR J 2002; 43: 207-213.

9. Primosch R E, Brooks R. Influence of anesthetic flow rate delivered by the Wand Local Anaesthetic System on pain response to palatal injections. Am J Dent 2002; 15: 15-20.

10. Frankl S H, Shiere F R, Fogels H R. Should the parent remain within the dental operatory? J Dent Child 1962; 29: 150-163.

11. Tahmassebi J F, Nikolaou M, Duggal M S. A comparison of pain and anxiety associated with the administration of maxillary local analgesia with Wand and conventional technique. Eur Arch Paediatr Dent 2009; 10: 77-82.

12. Ram D, Peretz B. The assessment of pain sensation during local anesthesia using a computerized local anesthesia (Wand) and a conventional syringe. J Dent Child (Chic) 2003; 70: 130-133.

13. Yesilyurt C, Bulut G, Taşdemir T. Pain perception during inferior alveolar injection administered with the Wand or conventional syringe. Br Dent J 2008; 205: E10, discussion 258-259. 